\title{
Splenic Artery Embolization: Endovascular and Percutaneous Trans-Splenic Approach
}

\author{
Pankaj Banode ${ }^{1}$ Gaurav Sharma ${ }^{1}$ \\ ${ }^{1}$ Department of Interventional Radiology, DMIMS, Sawangi,
Wardha, Maharashtra, India
}

Int J Recent Surg Med Sci 2018;4:47-53

\begin{abstract}
Address for correspondence Gaurav Sharma, DMRD, DNB Radiology, Department of Interventional Radiology, DMIMS, Sawangi, Wardha 442001, Maharashtra, India (e-mail: dr.gauravsharmabaloo@gmail.com).
\end{abstract}

\begin{abstract}
Keywords

- portal hypertension

- hypersplenism

- splenic artery embolization

- splenic artery pseudoaneurysm

- percutaneous embolization

Background Splenic artery embolization is becoming a widely acceptable procedure for nonsurgical management in many clinical settings such as pseudoaneurysms or to salvage splenic functions in portal hypertension, hypersplenism, and secondary prophylaxis in variceal hemorrhage. Various published meta-analyses of splenic artery embolization found an overall success rate of $90 \%$ in vascular abnormality.

Methods Retrospective study of all splenic artery embolization procedures in 1 year (January 2017-2018) to analyze various indications, procedure technique, and its modification as well as outcome measurement and complications.

Results Total 16 splenic artery embolization procedures were performed including one case of percutaneous trans-splenic glue embolization in 1-year duration.

Procedure Indication It included pseudoaneurysm ( $n=7,44 \%)$; trauma $(n=1,6 \%$; adjuvant to surgical splenectomy in hypersplenism $(n=3,18 \%)$; secondary prophylaxis in portal hypertension, portosystemic varices with/without reduced platelet counts, or ascites $(n=4,25 \%)$; and Budd-Chiari syndrome $(n=1,6 \%)$.Total $13(81 \%)$ procedures were elective, whereas emergency embolization was performed in 3 (18.7\%). Endovascular technical success was $93.7 \%(n=15)$ cases. Percutaneous trans-splenic embolization was performed in one $(6 \%)$ case in which access to bleeding point was not possible. The embolic agents included combination of metallic coils $(n=14,87.5 \%)$, Gelfoam ( $n=6,37.5 \%)$, polyvinyl alcohol (PVA) particles $(n=5,31 \%)$, and cyanoacrylate glue $(n=3,18.7 \%)$. Complication rate was low with development of splenic abscess with infected pyothorax in one (6\%) case of hypersplenism requiring pigtail drainage with uneventful full recovery.

Conclusion Splenic artery embolization provides safer nonsurgical options in management of cases such as trauma, hypersplenism, portal hypertension for control of hemorrhage and preservation of splenic function. Endovascular embolization facilitates complete exclusion of pseudoaneurysms in majority with percutaneous splenic embolization using glue or coils as a safer alternative where endovascular access to bleeding vessels is difficult.
\end{abstract}

\section{Introduction}

Splenic artery embolization (SAE) is becoming a widely acceptable procedure to treat diseases affecting the splenic artery or relating to splenic vascularity such as pseudoaneurysm, trauma, and hypersplenism. In appropriate clinical settings, these interventions provide a solid alternative to open surgery like in pseudoaneurysm. Its use is increasing to salvage splenic function in trauma and hypersplenism and to improve liver function in portal hypertension 
by reducing portal pressure and its sequelae. Its use is also highlighted in secondary prevention of variceal hemorrhage and as a preoperative adjuvant therapy to reduce intraoperative blood loss. Splenic artery pseudoaneurysm is the most common clinical issue, especially in cases of pancreatitis with risk of rupture of 3 to $10 \%$ carrying mortality of 20 to $100 \%{ }^{1}$ Percutaneous trans-splenic embolization is a safer alternative to endovascular approach in cases with tortuous vascular anatomy, making it difficult to access pseudoaneurysm neck or in large sized aneurysm. ${ }^{2}$

This study was undertaken to analyze in detail the various indications, procedure techniques and modification, outcome measurement, and safety and complications of all SAE procedures done in 1-year duration in rural-based catheter laboratory setup.

\section{Materials and Methods}

This study involved retrospective analysis of all SAE procedures in 1-year duration between January 2017 and 2018 at a rural-based hospital. Chart review was used to collect patient demographic details, imaging data such as computed tomographic (CT) scan, laboratory tests, procedure indications, technique, clinical outcomes, and complications.

Endovascular embolization procedure was performed in catheter laboratory on DSA Unit, Philips Allura Xper FD 20 DSA, after all routine hematologic tests, preprocedure antibiotics, and pneumococcal vaccine in selected cases.

Routine right transfemoral route was used in 12 cases, whereas radial route was accessed in 3 cases. A 5F visceral catheter Cobra/SIM1 was used for initial celiac angiogram to delineate splenic artery anatomy, and further procedures were completed using coaxial micro catheter (2.8F Progreat and Cantata) system with use of 0.018/0.035 metallic micro nester or nester coils in combination with other embolic agents such as glue and polyvinyl alcohol (PVA).

Percutaneous trans-splenic direct pseudoaneurysmal sac puncture as a modified technique was performed using 25G $15 \mathrm{~cm}$ Chiba needle under ultrasound (USG) guidance, and embolization was done using $20 \%$ glue under fluoroscopic guidance in one case in which endovascular approach failed due to difficult tortuous vessel.

The measured outcome for this study included technical success, clinical efficacy, and complication according to the Society of Interventional Radiology standards of practice committee's classification. ${ }^{3}$

\section{Results}

Total 16 SAE procedures were identified for the retrospective analysis with 11 (69\%) male and 5 (31\%) female cohorts having age range of 21 to 50 years.

Comorbid associations included diabetes $(n=2,12.5 \%)$, cirrhosis-portal hypertension $(n=6,37.5 \%)$, and pancreatitis $(n=7,43.7 \%)$. Total $13(81 \%)$ procedures were elective, whereas emergency embolization was performed in 3 (18.7\%).

Procedure indication included pseudoaneurysm $(n=7,44 \%)$; trauma ( $n=1,6 \%$ ); adjuvant to surgical splenectomy in hypersplenism ( $n=3,18 \%)$; secondary prophylaxis in portal hypertension, portosystemic varices with/without reduced platelet counts, or ascites ( $n=4,25 \%)$; and Budd-Chiari syndrome $(n=1,6 \%)$.

Endovascular technical success was $93.7 \%(n=15)$ cases with complete sac exclusion in 6 (86\%) patients with pseudoaneurysm. Percutaneous trans-splenic embolization was performed in one patient in whom access to bleeding point was not possible (-Fig. 1).

No repeat or second embolization setting was required in any case.

The embolic agents included combination of metallic coils ( $n=14,87.5 \%)$, Gelfoam ( $n=6,37.5 \%)$, PVA particles $(n=5,31 \%)$, and cyanoacrylate glue ( $n=3,18.7 \%)$ used mainly through coaxial micro catheter delivery system (Progreat/ Cantata).

Average number of coils used was two per case with or without combination of glue/PVA. Maximum number of coils used was five in one case. Complete glue embolization was performed in one patient with major active gastrointestinal (GI) bleeding with very poor general condition having multiple pseudoaneurysm involving more than $75 \%$ of splenic artery. Complete aneurysm exclusion was achieved in six (86\%) cases through endovascular approach (-Fig. 2).

Endovascular partial SAE as an adjuvant to splenectomy was performed in three $(18.7 \%)$ cases in which mid-distal segment was embolized and 50 to $70 \%$ splenic parenchymal infarct was achieved at 1-month follow-up.

Partial SAE was performed in five (31\%) cases of portal hypertension with portosystemic varices. At 3-month follow-up, no major GI bleeding was reported with overall improvement in liver function, platelet counts, and reduction in ascites and pleural effusion. More definitive further hepatic interventions (diversion) were advised in three cases; however, they were declined by patients.

Special mention of one 21-year-old woman with BuddChiari syndrome diagnosed with hepatic adenoma, large splenic artery pseudoaneurysm, and splenorenal varices with reduced platelet counts requiring total five nester coils to exclude the aneurysm. On follow-up, no variceal bleeding occurs with improvement in her blood counts and reduction in ascites and pleural effusion (- Fig. 3 ).

Complication rate was low with minor post-embolization syndrome symptoms. Development of splenic abscess with infected pyothorax was seen in one (6\%) case of hypersplenism requiring pigtail drainage with uneventful full recovery. One patient died post-embolization on sixth day due to comorbid condition unrelated to procedure.

Procedure details are described in - Table $\mathbf{1}$.

\section{Discussion}

SAE has been performed as an adjunct to nonsurgical treatment of both vascular and nonvascular splenic-related conditions. The first SAE was performed in 1973 for recurrent GI hemorrhage from esophageal varices. ${ }^{4}$ With the advancement in technology and improved sterile technique, Spigos et al performed first partial SAE to treat recurrent 


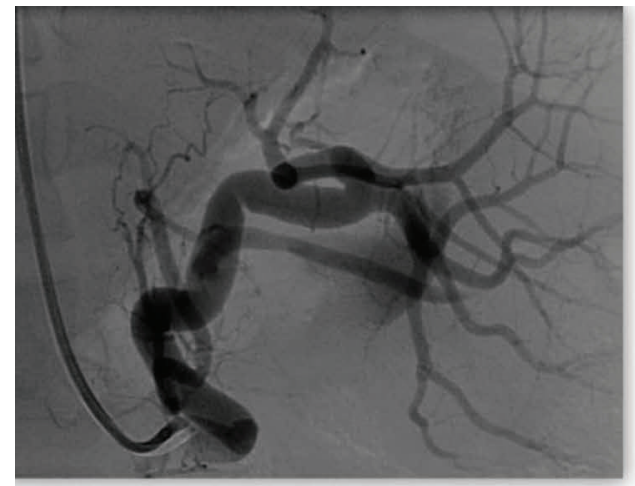

$\mathbf{A}$

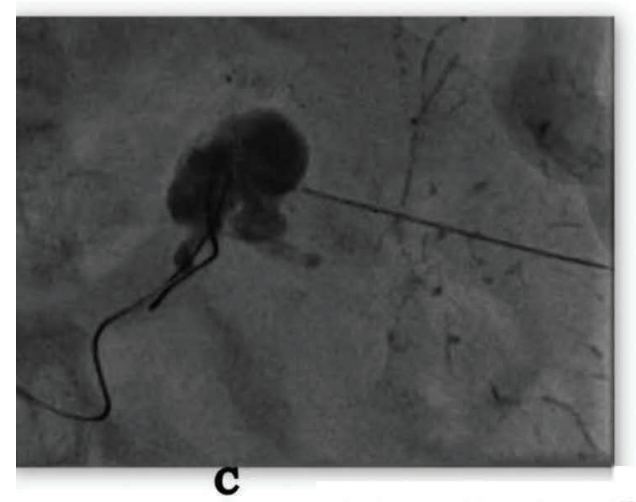

Fig. 1 (A-D) Percutaneous trans-splenic glue embolization of large splenic pseudoaneurysm after failed endovascular access.

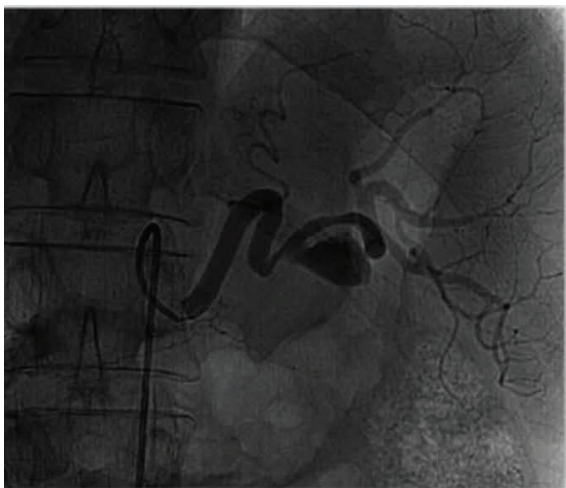

A

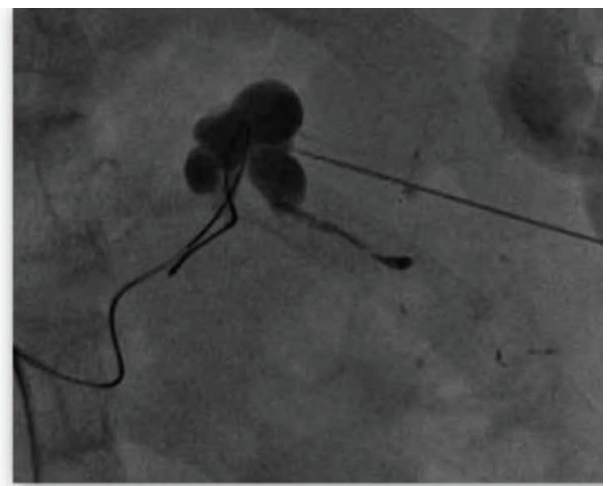

B

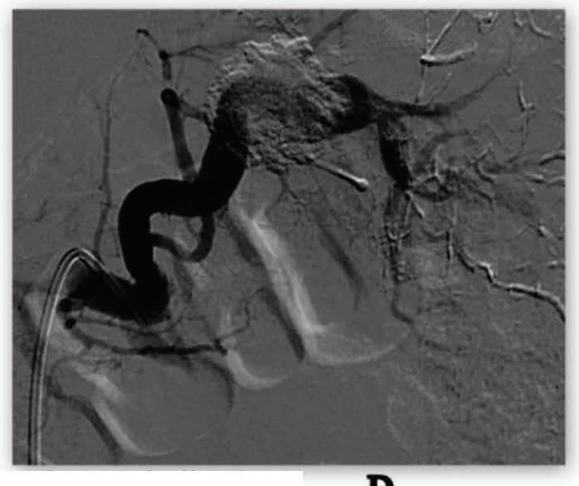

D

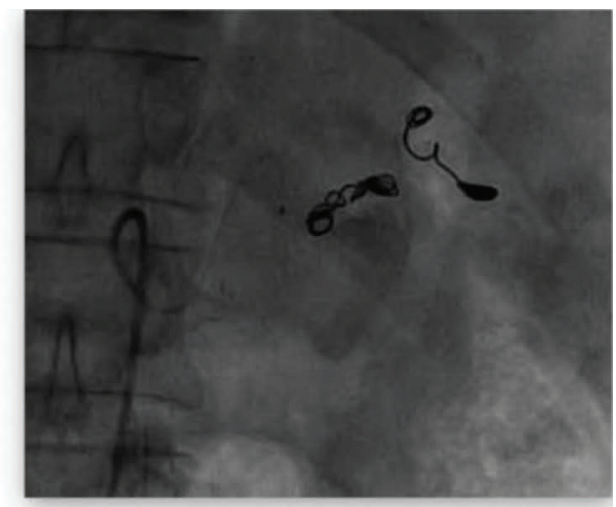

B

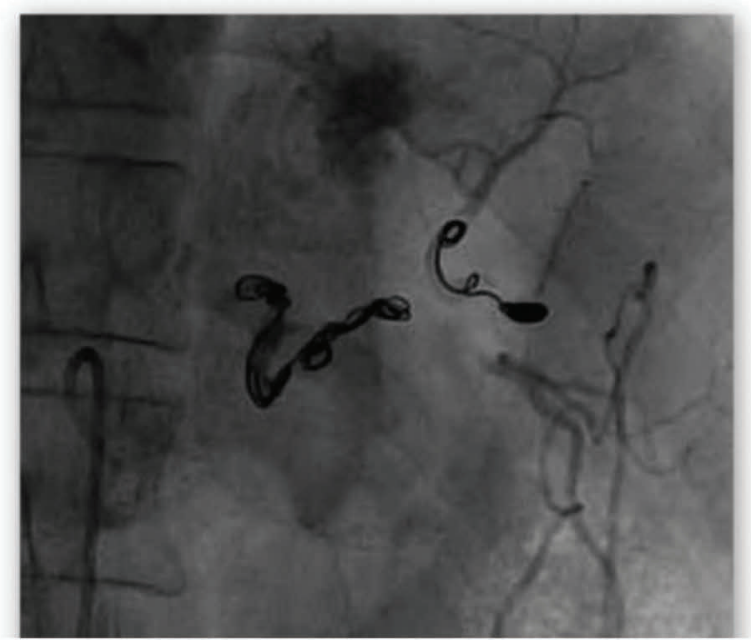

C

Fig. 2 (A-C) Distal splenic artery pseudoaneurysm with coil migration and final sandwich embolization. 


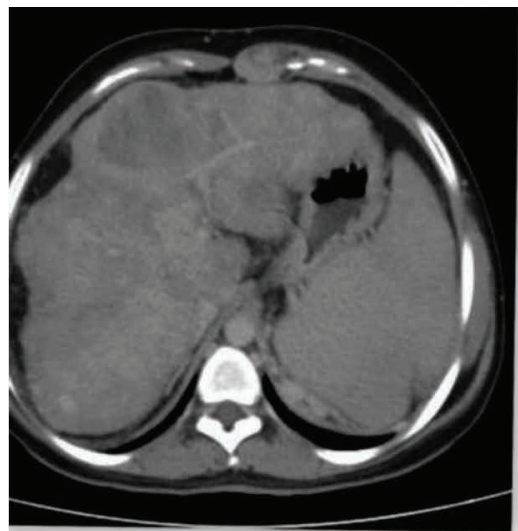

\section{A: Budd Chiari Syndrome with hepetic Adenoma}

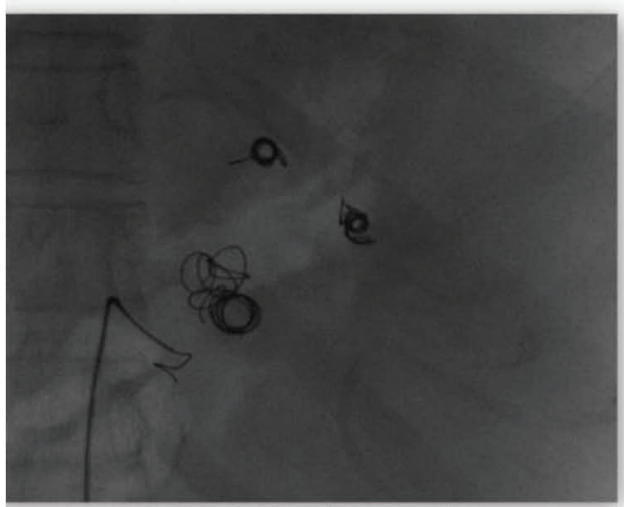

C: Total 5 coils used

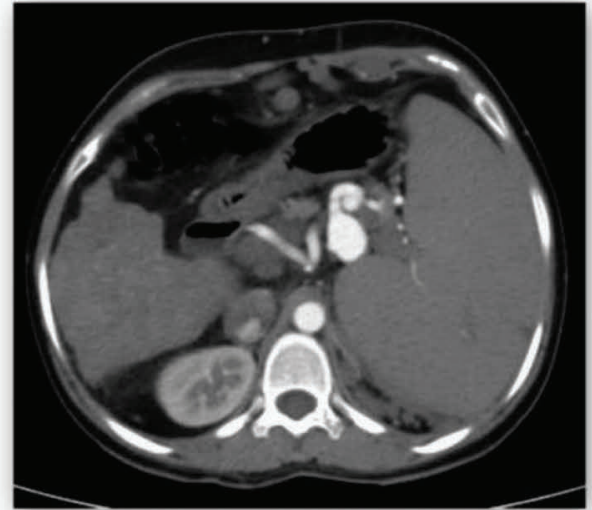

B: Splenic artery Aneurysm

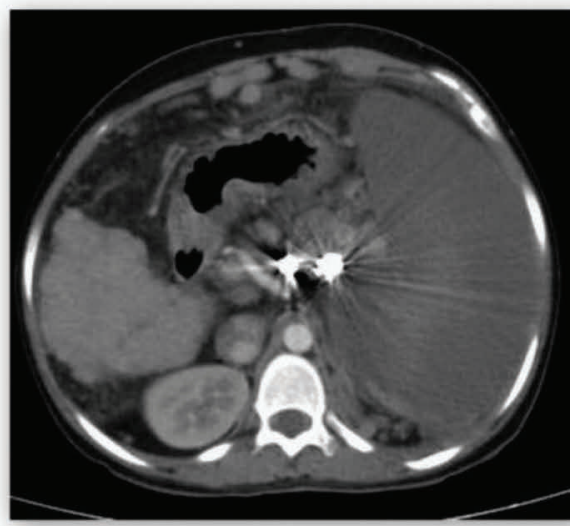

D: Post Coil Status

Fig. 3 (A-D) A 24-year-old woman with Budd-Chiari syndrome, hepatic adenoma, and splenic artery aneurysm requiring maximum numbers of coils with follow-up CT of abdomen.

Table 1 Procedure details

\begin{tabular}{|c|c|c|c|c|c|}
\hline$n=16$ & Pseudoaneurysms & $\begin{array}{l}\text { Splenic trauma } \\
\text { (-Fig. } 4 \text { ) }\end{array}$ & Hypersplenism & PHT-cirrhosis & $\begin{array}{l}\text { Budd-Chiari } \\
\text { syndrome }\end{array}$ \\
\hline No. of cases & $\begin{array}{l}7(44 \%) \\
\text { Distal: } 5(72 \%) \\
\text { Proximal: } 2(28.6 \%)\end{array}$ & $1(6 \%)$ & $3(18 \%)$ & $4(25 \%)$ & $1(6 \%)$ \\
\hline Sex & $\mathrm{M}(6), \mathrm{F}(1)$ & $M(1)$ & $\mathrm{M}(1), \mathrm{F}(2)$ & $\mathrm{M}(3), \mathrm{F}(1)$ & $F(1)$ \\
\hline Mean age (y) & 37 & 45 & 42 & 43 & 21 \\
\hline Embolic agent & $\begin{array}{l}2 \text { coils: } 3 \\
3 \text { coils: } 1 \\
3 \text { coils + glue: } 1 \\
\text { Glue: } 2^{\text {a }}\end{array}$ & 2 coils & 5 coils + PVA & $\begin{array}{l}4 \text { coils + PVA: } 3 \\
3 \text { coils + PVA: } 1\end{array}$ & 5 coils \\
\hline $\begin{array}{l}\text { Follow-up at } 1 \\
\text { and } 3 \text { months }\end{array}$ & $\begin{array}{l}\text { Complete occlusion in } \\
6^{\text {b }} \text { without any recur- } \\
\text { rent bleeding }\end{array}$ & $\begin{array}{l}\text { Stable without } \\
\text { any surgical } \\
\text { intervention }\end{array}$ & $\begin{array}{l}\text { Stable hematologic } \\
\text { indices in all } 3\end{array}$ & $\begin{array}{l}\text { No major } \\
\text { bleeding } \\
\text { Better liver } \\
\text { function } \\
\text { Red ascites }\end{array}$ & $\begin{array}{l}\text { Complete occlu- } \\
\text { sion of pseudo } \\
\text { with improved } \\
\text { blood profile }\end{array}$ \\
\hline Complication & $\begin{array}{l}\text { Minor puncture } \\
\text { hematoma: } 1\end{array}$ & - & $\begin{array}{l}\text { Splenic abscess, } \\
\text { pyothorax: } 1\end{array}$ & - & $\begin{array}{l}\text { Increased pleural } \\
\text { effusion }\end{array}$ \\
\hline Tech success & $86 \%$ & $100 \%$ & $100 \%$ & $100 \%$ & $100 \%$ \\
\hline
\end{tabular}

Abbreviations: F, female; M, male; PHT, portal hypertension; PVA, polyvinyl alcohol.

ane case underwent percutaneous trans-splenic glue embolization.

${ }^{b}$ One patient died due to comorbid condition unrelated to procedure. 


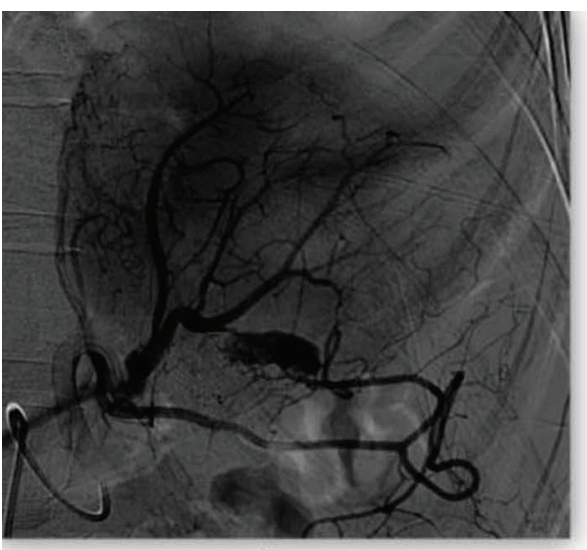

$\mathbf{A}$

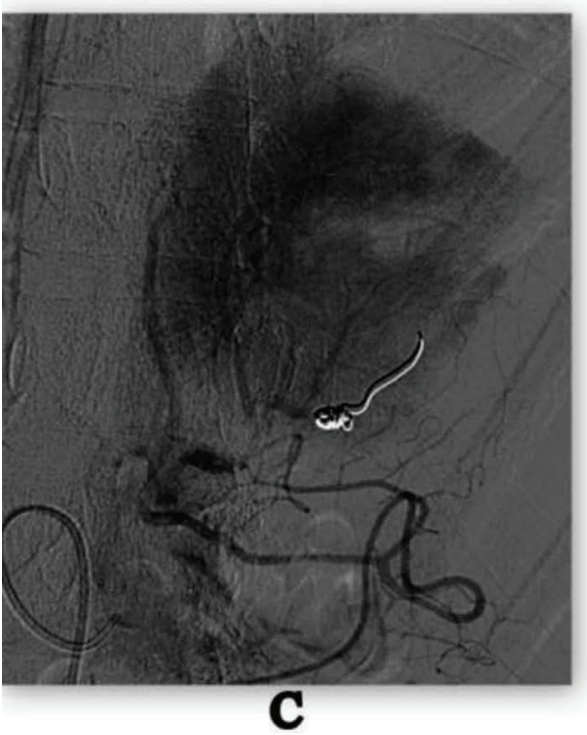

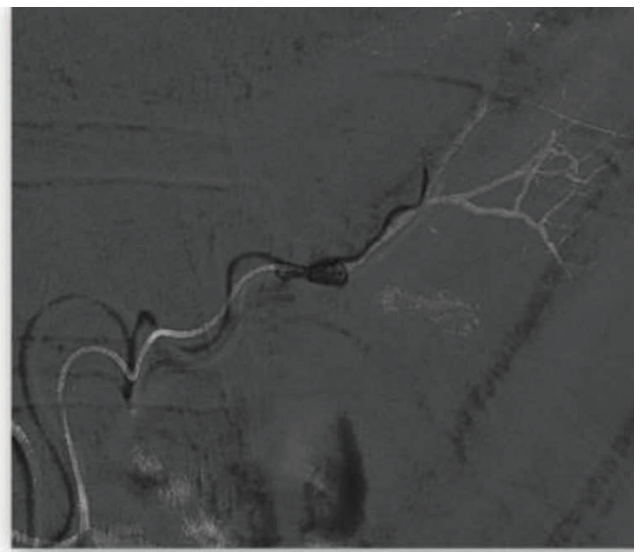

B

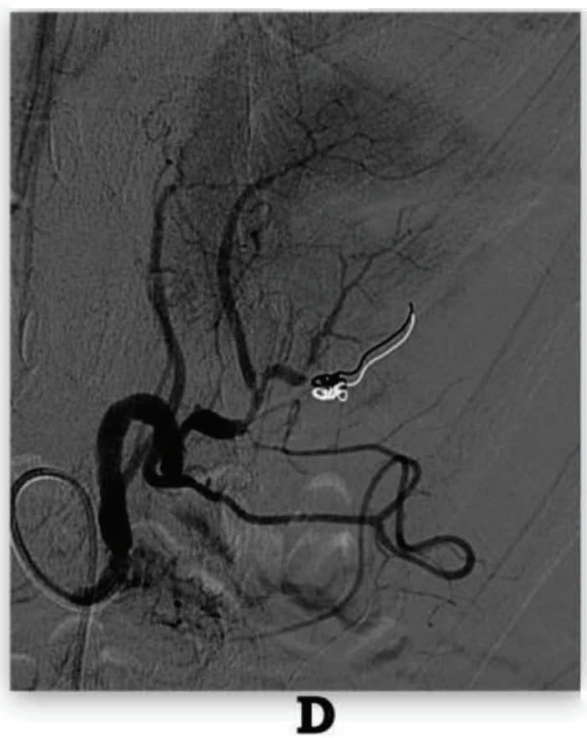

Fig. 4 (A-D) Splenic trauma with pseudoaneurysm and active contrast extravasation treated with coil embolization.

esophageal hemorrhage in 1979. Recent data analysis has proven the utility of SAE with failure rate of only $15 \%$ in management of blunt splenic trauma, while having $90 \%$ success rate in treatment of splenic artery aneurysms and pseudoaneurysms. ${ }^{5}$ Our study outcome is comparable with various studies with high degree of technical success (94\%).

Splenic artery aneurysms and pseudoaneurysms are the most common visceral artery aneurysms (60\%), followed by the hepatic artery (20\%), superior mesenteric artery (SMA) (5.9\%), and celiac artery (4\%). Rupture of splenic aneurysm is uncommon and asymptomatic, with reported rate of rupture ranging between 3 and $10 \%$, but if rupture occurs, it is fatal in 20 to $100 \% .{ }^{6}$ When compared with surgical intervention, endovascular transcatheter embolization has significantly lower morbidity and mortality. ${ }^{7}$ Taori et $\mathrm{al}^{8}$ successfully treated 16 patients with splenic artery aneurysm via transcatheter technique with $100 \%$ success rate. They also emphasized the importance of technique. In presence of good collaterals (gastric, pancreatic), both distal and proximal segments should be embolized (sandwich technique). Proximal embolization is considered in case of poor or no collaterals. Embolization using coils has disadvantage of recanalization due to poor sac packing. This has led to use of supplementary embolic agents such as glue, thrombin, or PVA particles. Our study demonstrated a $94 \%$ technical success in pseudoaneurysm embolization without any major complication or recurrence at 3-month follow-up. Supplementary embolic materials in combination with metallic coils were used in three cases.

Direct puncture and percutaneous embolization technique are commonly used for peripheral and visceral artery pseudoaneurysm embolization with high safety and success rate. ${ }^{2}$ These techniques are used in angiographic scenario with torturous vessel course, acute angle, and arterial feeder spasm leading to difficult or failed cannulation of aneurysm sac, performed under USG/fluoroscopic guidance. Coils, thrombin, glue, or PVA particles all can be used as embolic agent alone or in combination. ${ }^{9}$

In this retrospective cohort, successful endovascular embolization was achieved in six out of seven patients with pseudoaneurysm. In one patient with difficult failed cannulation on two occasions, direct trans-splenic sac puncture under USG guidance was performed using 22G Chiba needle and glue embolization was done under continuous 
angiographic control. Complete sac exclusion was achieved without any untoward complication.

Hypersplenism is clinically characterized by thrombocytopenia, leucopoenia, splenomegaly secondary to increased pooling or destruction of blood cells commonly seen in cirrhosis, and portal hypertension. Partial SAE in which portion of splenic parenchyma is left viable is a safer and effective alternative to surgery. This can also be used as adjuvant bridge therapy for interval splenectomy. Postembolization, these patients showed reduction in ascites with pleural effusion and overall improvement in liver function with reduced size of portal and splenic vein. ${ }^{10}$

This cohort had three cases of hypersplenism undergone partial SAE with improved blood counts and liver function at 3-month follow-up. One patient developed splenic abscess and left pyothorax requiring pigtail drainage with uneventful complete recovery at 2 months.

Haan et $\mathrm{al}^{11}$ and Bessoud and Denys ${ }^{12}$ analyzed the role of SAE in hemodynamically stable patients having up to GIII splenic laceration and active arterial injury on CT, and found greater than $90 \%$ success rate in nonsurgical salvage of splenic function avoiding surgical complications and post-splenectomy risk of infections. Our study had one case of splenic laceration with traumatic pseudoaneurysm at distal segment with active contrast extravasation treated successfully with nester coils in combination with 300 to 500 PVA particles. Generally, embolization technique depends on type of injury. In case of arterial injury, distal coil embolization with selective exclusion of any pseudoaneurysm sac is preferred. Patients with increase risk of splenic rupture should undergo proximal embolization to reduce the pressure in the splenic parenchyma. Proximal embolization decreases the volume of splenic arterial blood flow, causing relative hypotension in splenic bed that allows repair without infarction. ${ }^{13}$

Recently there has been an increased tendency toward a more conservative noninvasive management in patients with cirrhosis and portal hypertension with variceal bleeding, hypersplenism, and hepatogenic ascites as its complication. The combination of varices and low platelet counts puts this patient at increased risk of life-threatening hemorrhage. Koconic et al $^{14}$ reviewed all PubMed literature from 1973 to 2005 on use of partial SAE in patients with portal hypertension and found partial SAE to be efficacious in controlling variceal bleeding, improving hematologic indices and hepatic function, and reducing the severity of hepatic encephalopathy. Our retrospective analysis also supports these observations in five patients with portal hypertension who underwent mid-distal segment SAE with satisfactory improvement in overall liver function. In three cases, definitive diversion treatment (transjugular intrahepatic portosystemic shunt/ direct intrahepatic portacaval shunt [TIPS/DIPS]) was advised; however, it was refused by the patients in view of good response.

Gaba et $\mathrm{a}^{15}$ evaluated in details the various prognostic factors to the clinical outcome of SAE. Advanced age, Child Pugh class C, postprocedural thrombocytopenia, postprocedural hydrothorax-splenic abscess, poor renal reserve, and hemodynamic instability are important predictors to the final clinical outcomes. ${ }^{13,15}$

In this cohort, complications rates were very low with development of splenic abscess with pyothorax as major complication noted in one patient only, which was managed successfully conservatively.

Important limitation of this study was that it was a retrospective single-center study with low study population.

\section{Conclusion}

Although this study was performed for 1-year duration only, several SAE procedures were performed at this center in the past 15 years with promising results in various clinical conditions such as trauma, vascular entities, portal hypertension, and hypersplenism. Further research is required to assert the role interventional radiology in various splenic interventions, with focus on technical improvement and defining the prognostic factors.

\section{Conflict of Interest}

None.

\section{References}

1 Yoon T, Kwon T, Kwon H, Han Y, Cho Y. Transcatheter arterial embolization of splenic artery aneurysms: a single-center experience. Vasc Spec Int 2014;30(4):120-124

2 Shrivastava A, Rampal JS, Reddy DN, Rao GV. Direct needle puncture and embolization of splenic artery pseudoaneurysm in case of chronic atrophic calcified pancreatitis. Pol J Radiol 2016;81:462-464

3 Angle JF, Siddiqi NH, Wallace MJ, et al; Society of Interventional Radiology Standards of Practice Committee. Quality improvement guidelines for percutaneous transcatheter embolization: Society of Interventional Radiology Standards of Practice Committee. J Vasc Interv Radiol 2010;21(10):1479-1486

4 Ahuja C, Farsad K, Chadha M. An overview of splenic embolization. AJR Am J Roentgenol 2015;205(4):720-725

5 Loffroy R, Guiu B, Cercueil JP, et al. Transcatheter arterial embolization of splenic artery aneurysms and pseudoaneurysms: short- and long-term results. Ann Vasc Surg 2008;22(5):618-626

6 Ha JF, Phillips M, Faulkner K. Splenic artery aneurysm rupture in pregnancy. Eur J Obstet Gynecol Reprod Biol 2009;146(2):133-137

7 Hogendoorn W, Lavida A, Hunink MG, et al. Open repair, endovascular repair, and conservative management of true splenic artery aneurysms. J Vasc Surg 2014;60(6):1667-76.e1

8 Taori K, Rathod J, Disawal A, et al. Endovascular embolization of splenic pseudoaneurysms complicating pancreatitis using micro coils: case series. OJRad 2013;3(1):33-40

9 Madhusudhan KS, Venkatesh HA, Gamanagatti S, Garg P, Srivastava DN. Interventional radiology in the management of visceral artery pseudoaneurysm a review of techniques and embolic materials. Korean J Radiol 2016;17(3):351-363

10 Abdella HM, Abd-El-Moez AT, Abu El-Maaty ME, Helmy AZ. Role of partial splenic arterial embolization for hypersplenism in patients with liver cirrhosis and thrombocytopenia. Indian J Gastroenterol 2010;29(2):59-61

11 Haan J, Scott J, Boyd-Kranis RL, Ho S, Kramer M, Scalea TM. Admission angiography for blunt splenic injury: advantages and pitfalls. J Trauma 2001;51(6):1161-1165 
12 Bessoud B, Denys A. Main splenic artery embolization using coils in blunt splenic injuries: effects on the intrasplenic blood pressure. Eur Radiol 2004;14(9):1718-1719

13 Madoff DC, Denys A, Wallace MJ, et al. Splenic arterial interventions: anatomy, indications, technical considerations, and potential complications. Radiographics 2005;25(Suppl 1): S191-S211
14 Koconis KG, Singh H, Soares G. Partial splenic embolization in the treatment of patients with portal hypertension: a review of the English language literature. J Vasc Interv Radiol 2007;18(4):463-481

15 Gaba RC, Katz JR, Parvinian A, et al. Splenic artery embolization: a single center experience on the safety, efficacy, and clinical outcomes. Diagn Interv Radiol 2013;19(1):49-55 